\title{
Managing bowel obstruction in
}

\section{ovarian cancer using a percutaneous endoscopic gastrostomy (PEG) tube}

\author{
By Lynne Jolicoeur and Wylam Faught
}

\section{Abstract}

An estimated 2,500 women were diagnosed with and 1,500 died from ovarian cancer in Canada in 2002. Up to $42 \%$ of patients in the palliative phase develop a malignant bowel obstruction. Options for management include medical therapy, surgery, and/or a percutaneous endoscopic gastrostomy (PEG) tube.

The objective of this quality improvement study was to: 1) examine if successful palliation was achieved using a PEG tube, and 2) identify opportunities to improve the quality of nursing care provided. A retrospective review of 24 patient records revealed that $75 \%$ did not have nausea/vomiting by time of discharge; $92 \%$ resumed a clear fluid diet; $83 \%$ were discharged from the acute care setting; and $70 \%$ did not require re-admission. A PEG tube may effectively palliate women with non-operable bowel obstruction in advanced/recurrent cancer of the ovary. Opportunities for improving care are presented.

Ovarian cancer is the most common cause of death from gynecological cancers and is the fifth leading cause of cancer death among women in Canada and the United States. It is estimated that in Canada in 2002, 2,500 women were diagnosed with and 1,500 died of ovarian cancer (National Cancer Institute of Canada, 2002). Although the relative five-year survival rates improved significantly from the mid-1970s to the mid-1990s, survival remains dismally low at $44 \%$ for all stages. Because early ovarian cancer is typically asymptomatic, only $25 \%$ of patients are diagnosed with localized disease. Women diagnosed with stage three and four disease have a 10 to $30 \%$ fiveyear survival (DiScia \& Creasman, 2002).

The literature suggests that $5.5 \%$ to $25 \%$ of women with ovarian cancer develop a malignant bowel obstruction. Up to $42 \%$ of women with terminal ovarian cancer will develop this condition (Ripomonti, 1994). Bowel obstruction is often the first manifestation of recurrent ovarian cancer. In such cases, bowel obstruction indicates a poor prognosis and is often a pre-terminal event (Beattie, Leonard, \& Smyth, 1989). The goal of treatment is to relieve symptoms and enhance quality of life, as the cancer will likely not respond to further therapy and the obstruction often may not resolve.

In ovarian cancer, the pathophysiology of the obstruction is related to extrinsic compression on the bowel and by carcinomatous ileus in which bowel motility is compromised (Cain, Wenzel, Monk, \& Cella, 1998). The most common site of obstruction is the small bowel (Feuer, Broadley, \& Barton, 2000).

Options for management of bowel obstruction include: laparotomy with surgical bypass, pharmaceutical management, percutaneous endoscopic gastrostomy (PEG) or nasogastric (N/G) drainage, and chemotherapy. However, the optimal management of bowel obstruction in recurrent ovarian cancer remains controversial.

Surgery remains the treatment that offers the longest survival outcome in the management of bowel obstruction, but considerable attention is needed to determine which patients will benefit from surgery (Gadducci et al., 1998; Jong, Sturgeon, \& Jamieson, 1995; Ripomonti, 1994; van Ooijen, van der Burg, Planting, Siersema, \&
Wiggers, 1993). The scoring system identified by Krebs and Goplerud offers reliable eligibility criteria (cited in Gadducci et al.). It is based on the variables of patient's age, nutritional status, tumour status, amount of ascites, and previous treatment history of chemotherapy and radiotherapy. Jong et al. conducted a retrospective chart review in which successful palliation was achieved in $51 \%$ of patients with the absence of four prognostic factors: palpable abdominal and pelvic mass, ascites exceeding three litres, multiple obstructive sites, and preoperative weight loss of greater than nine kilograms. Similar criteria are used to identify which patients should be counselled about surgery at our centre. The use of such criteria may reduce unnecessary procedures, as well as lower patient morbidity.

In addition to surgery, chemotherapy with the goal of cancer control and relief of the bowel obstruction is a potential therapeutic option. Abu-Rustum, Barakat, Venkatramann, and Spriggs (1997) concluded in their study that chemotherapy was ineffective in restoring bowel function in heavily pre-treated women with recurrent ovarian cancer. They recommended that this treatment should be limited to chemotherapy-naïve patients.

When surgery and chemotherapy are not practical therapeutic options, health care professionals are left to guide patients and their families in decision-making about how best to control the symptoms of bowel obstruction and to optimize quality of life.

Symptom control of bowel obstruction in terminal ovarian cancer using a pharmaceutical approach alone has been described in the literature (Baumrucker, 1998; Gadducci et al., 1998; Mangili et al., 1996; Ripomonti, 1994) and has been used successfully in the hospice setting. Fainsinger, Spachynski, Hanson, and Bruera (1994) conducted a retrospective chart review of patients in their palliative care unit admitted with bowel obstruction. The medication regimen included: Dexamethasone with a mean dose of $40 \mathrm{mg} /$ day $(8-60 \mathrm{mg})$; Methoclopramide $10 \mathrm{mg} \mathrm{q} 4 \mathrm{~h}$, and increased to $60-120 \mathrm{mg} /$ day in four of 15 patients. Other drugs used included Dimenhydrate, Haloperidol, Domperidone, and Buscopan. Twenty-six per cent of the patients in their palliative care unit were managed with a PEG tube. They concluded that malignant bowel obstruction can be successfully managed with minimal use of NG and IV hydration. A wide variety of regimens are reported in the literature, but all agree that the regimen must be tailored to the individual patient (Baumrucker; Gadducci et al.; Mangili et al.; Ripomonti).

Some researchers (e.g., Khoo et al, 1994; Mangili et al., 1996; Mercadante, Spoldi, Caraceni, Maddaloni, \& Simonetti, 1993) found that Octreotide was effective in controlling the amount of gastric fluid produced and in improving symptoms of bowel obstruction. Sixtytwo per cent of patients in Mangili et al.'s study were able to be discharged home.

Lynne Jolicoeur, RN, BScN, CON $(C)$, is a Gynecologic-Oncology APN (intern), Ottawa Hospital, General Campus, Ottawa, ON Wylam Faught, MD, FRCSC, is Professor and Chair, Department of Obstetrics and Gynecology, Chief, Women's Health Program, Royal Alexandra Hospital, Edmonton, Alberta. 
The use of a percutaneous endoscopic gastrostomy (PEG) tube has been recommended in the literature as a way of managing malignant bowel obstruction when surgery is not indicated/successful (Frankel Kelvin \& Scagliola, 1998; van Ooijen et al., 1993), or when a pharmaceutical approach is not effective in the hospice setting (Baumrucker, 1998; Ripomonti, 1994). The PEG tube has been reported as a cost-effective and safe method for gastric decompression when compared to a nasogastric tube and other gastrostomy tube (Adelson \& Kasowitz, 1993; Marks, Perkal, \& Schwartz, 1993). All of the available studies concluded that PEG was safe and demonstrated lower morbidity than an N/G or gastrostomy done by laparotomy.

Over the past three years, the approach at our centre to treating patients with bowel obstruction secondary to recurrent ovarian cancer has been to offer percutaneous endoscopic gastrostomy (PEG) drainage in the home or hospice to control the symptoms of obstruction. Patients are initially managed conservatively with nasogastric suction, intravenous hydration, corticosteroids and, for selected patients, prokinetic agents. All patients are initially considered for bypass surgery. When surgical intervention is not considered appropriate by the surgeon, a consultation with the gastroenterology service is requested for PEG tube placement. Gastrostomy tube placement is performed in the endoscopic suite, patients are pre-medicated, the site is verified for absence of metastasis, and the skin is prepped. The PEG tube is passed through the mouth and out the abdominal wound in a smooth single motion, a technique described by Marks et al. (1993).

Following the patient's return to the unit, the PEG tube is connected to a Foley collection bag for gravity drainage. The PEG tube is clamped for 30 minutes prior to oral medications and meals, or for as long as the patient does not experience nausea, vomiting, and/or abdominal bloating. The PEG tube can be connected to intermittent or continuous suction if gravity drainage is not sufficient to control nausea and vomiting. Patients are started on a clear fluid diet initially. The diet is then increased to liquid, pureed, and finally low-residue as tolerated. Oral intake is initially limited to fluid that can pass through the PEG tube. Once symptom control has improved, the PEG tube is kept clamped for increasingly longer periods of time. If this is welltolerated, the diet can be augmented to a low-residue diet. For patients whose bowel function returns, the PEG tube is left clamped and is flushed three to four times per day to maintain patency. The tube can also be accessed for oral suspension medications. It is not removed since re-obstructive symptoms are anticipated (Feuer et al., 2000).

Given the frequent use of PEG tubes in our facility to alleviate bowel obstruction in women with ovarian cancer, we designed a quality improvement study with two main objectives: 1) to explore whether or not successful symptom control was achieved when using a PEG tube in patients with recurrent ovarian/peritoneal cancer and bowel obstruction, and 2) to identify opportunities to improve the quality of nursing care provided to these women.

\section{Methodology}

The authors conducted a retrospective chart review involving all patients with bowel obstruction who were managed with a PEG tube between 1996 and 1999. At the time of the study, ethics approval was not required to conduct quality improvement retrospective chart reviews. The records of 32 patients were identified from the gynecologic oncology service patient information system. A total of eight patients were excluded from the study, four because the PEG tube insertion was not successful due to the presence of peritoneal metastasis in the gastric area and/or ascites present, and an additional four because their diagnosis was not ovarian or peritoneal cancer. The categories of data extracted were demographics, history of prior surgery and chemotherapy, prior admission for bowel obstruction, site of obstruction, length of stay, symptoms on discharge, location of discharge, diet on discharge, other methods used to manage the bowel obstruction, number of re-admissions post-PEG, date of death, and location of death.
Bowel obstruction was diagnosed in all patients based on clinical presentation and radiologic investigations. Patients were initially managed conservatively as previously described. All patients were evaluated for, but did not have a laparotomy. After consultation with the gastroenterology service, a PEG tube had been placed for palliation.

Successful symptom control was defined as: the elimination of obstructive symptoms (nausea, vomiting, and abdominal cramps/pain), the ability of the patient to resume at least a clear fluid diet, the absence of PEG tube complications, the patient's ability to be discharged from the acute care facility, and no re-admission to hospital for bowel obstruction.

\section{Results}

All 24 patients included in this study were diagnosed with bowel obstruction; $88 \% \quad(n=21)$ also presented with a diagnosis of recurrent/progressive disease. Three patients (12\%) had obstructed within 30 days of their primary diagnosis. Seventy-nine per cent of patients $(n=19)$ had been surgically debulked and $92 \%(n=22)$ had received chemotherapy. Seventy-nine per cent of patients in this study presented with a small bowel obstruction. Fifty per cent had one or two prior admissions for a bowel obstruction and one patient had previously reported symptoms of impending bowel obstruction.

The mean time from admission to PEG tube insertion was 8.1 days with a mean length of stay of 15.1 days. Thirty-eight per cent $(n=9)$ had complications resulting from the PEG tube insertion. Three patients were treated for a PEG tube site infection. Five patients had leakage at the PEG site, one of whom complained of discomfort at the site; all were managed with the application of a barrier cream. One patient experienced extreme pain as a result of pneumoperitonitis secondary to the PEG tube insertion. The pain was managed with analgesia and the pneumoperitonitis resolved within 48 hours.

Seventy-nine per cent of patients $(n=19)$ were receiving concomitant medications on discharge (e.g., antiemetics, prokinetics, analgesics, and $\mathrm{H} 2$ receptor antagonist) to manage symptoms related to the bowel obstruction or the cancer progression. Seven patients (29\%) were discharged with parenteral hydration, either via hypodermoclysis or a central venous access device. Hydration was given overnight or continuously, based on the patient's oral intake and patient preference. Seven patients were discharged on some type of bowel laxative or enemas. Four patients $(17 \%)$ subsequently received palliative chemotherapy with the goal of decreasing the carcinomatosis to the bowel and relieving the obstruction. One patient was chemotherapy-naïve and received chemotherapy after PEG insertion and return of bowel function. The patient was alive 364 days post-PEG procedure. Three patients received chemotherapy with the goal of restoring bowel obstruction and increasing their length of survival. Two patients discontinued chemotherapy due to toxicity, while a third went on to receive six cycles of Topotecan and lived for 274 days post-PEG. Chemotherapy likely increased her length of survival, but she was not able to progress to a solid diet and she spent $20 \%$ of her time in hospital to control toxicity from chemotherapy and chronic obstructive symptoms. However, she lived to see the birth of her first grandchild.

At the time of discharge, $75 \%$ of patients were relieved of nausea and $88 \%$ no longer vomited; $17 \%$ of patients complained of abdominal cramping and abdominal bloating was experienced by only $17 \%$ of patients. By discharge, $92 \%$ of patients were able to resume some type of oral intake. Twenty-nine per cent of patients could tolerate clear fluids, $42 \%$ progressed to a full fluid diet and, in $21 \%$ of patients, the diet fluctuated from a full fluid to a low-residue diet.

The PEG tube was put to gravity drainage in $33 \%$ of patients and in $17 \%$ it was put on continuous suction due to persistent vomiting on only gravity drainage. However, no data were available related to clamping, gravity drainage, or suctioning of the PEG in $50 \%$ of the cases. 
Twenty patients (83\%) were discharged from the acute care setting. Fifty-eight per cent were discharged home with a primary caregiver. Care at home was arranged with a physician who was available to make house calls. Palliative nursing, which included shift nursing, was provided when needed and available. A quarter of patients were discharged to a hospice. Thirteen per cent died in hospital within 26 days of being admitted for their bowel obstruction and one patient was transferred to a community hospital close to home.

Of the 20 patients discharged from the acute care setting, 30\% $(n=6)$ required re-admission post-PEG insertion. A total of 20 readmissions were required; three patients were re-admitted more than once. Two re-admissions were due to PEG tube complications, 10 were for management of obstructive symptoms, and four readmissions were unrelated to either the PEG tube or to bowel obstruction. The first re-admission occurred on average within 21.7 days of the patient being discharged (range from five to 60 days). The median survival for patients post-PEG insertion was 42 days (five to 1226).

\section{Discussion}

Bowel obstruction, particularly of the small bowel, is a common event in recurrent/progressive ovarian cancer (Gadducci et al., 1998). One of the primary goals in managing bowel obstruction, on recurrence of disease, is symptom control and palliation. The present study demonstrated that insertion of a PEG tube contributed to symptom control, not including nausea, in $75 \%$ of patients. The severity of nausea and the impact on overall well-being of the patient could not be determined from the documentation. Overall, 65\% of patients did not have symptoms on discharge. Ninety-two per cent of patients resumed oral intake, $83 \%$ of patients were discharged from the acute care setting, and $70 \%$ did not require re-admission.

There is limited literature on PEG as it relates to the control of obstructive symptoms. Available results are often limited to outcomes such as survival post-PEG insertion and complications of PEG tube insertions. This current study provides additional information that may be useful to clinicians and also raises some areas for further investigation.

Symptom control could have been improved for our patients by optimizing some of their concomitant medications. While similar pharmaceutical management was used in our patients' care, we used smaller doses than have been reported in the literature (Baumrucker, 1998; Gadducci et al., 1998; Mangili et al., 1996; Ripomonti, 1994). Most of the literature on pharmaceutical management of bowel obstruction reflects a hospice setting where ongoing assessment and knowledge in palliative care is available to tailor the choice of drugs and dose to each patient. This kind of attention may be effective in the hospital setting as well.

Women with ovarian cancer often prefer to be at home because their performance status is often still good at the time of obstruction, however, they also need their symptoms to be controlled. PEG may be a good option for use in the community when managing symptoms related to malignant bowel obstruction in this population. In many cases, fears about dying of dehydration and starvation are expressed by these patients and their families. The ability to resume oral intake is often accepted as an indicator of successful symptom control in bowel obstruction and the rate of resumption of oral intake was significant in this study. These results suggest that PEG may enable patients to remain at home because it helps ensure that oral intake will be possible in the management of symptoms. However, we were unable to determine how much intake was absorbed into the gut and how much drained out via the PEG. The absence of vomiting is also an important indicator of symptom control in bowel obstruction. This was significant in our study, as more than two-thirds of patients did not vomit post-PEG.
A little more than one-third of patients in this current study had complications from the PEG. There is limited literature reported on complications of PEG, such as leakage and site infection requiring topical antibiotics. In one study, erythema and tenderness at the site were reported as the most frequent problems, but no incidence was reported (Malone, Koonce, Larson, Freedman, Carrasco, \& Saul, 1986). Hopkins, Roberts, and Morley (1987) reported a 50\% rate of site infections and Marks et al. (1993) reported no leakage or wound infection for which they used prophylactic antibiotics. Adelson and Kasowitz (1993) reported one case (7\%) of sepsis. Malone et al. (1986) reported pain in one patient $(10 \%)$ who required continual analgesia and one $(10 \%)$ patient had fever for 24 hours post-PEG insertion. It is difficult to establish if our overall complications' rate is comparable to the literature, but a rate of $4 \%(n=1)$ severe complications is infrequent. This is the only case of pneumoperitonitis in six years of experience using PEG tubes.

The sample of patients in this study who went on to receive chemotherapy is limited. While we support the conclusion of AbuRustum et al. (1997) that chemotherapy should be limited to chemotherapy-naïve patients, single agent chemotherapy is still administered to women who request it as part of aggressive management of their recurrent cancer and bowel obstruction. Practice in our setting has been to administer chemotherapy no earlier than two to three weeks post-discharge home from PEG insertions. These women and their families must have a good understanding of the limitations and risk of chemotherapy and the women must be able to tolerate at least a full-fluid diet, as well as have a relatively good performance status.

Other authors (e.g., Fainsinger et al., 1994; Gemlo et al., 1986; Ripomonti, 1994) either used CVL hydration in their patients or used hypodermoclysis overnight or continuously to prevent dehydration. Twenty-nine per cent of our patients were administered hydration to prevent weakness secondary to dehydration, or as a supportive measure for patients who feared dying of malnutrition and dehydration.

\section{Nursing implications}

Nurses play a significant role in providing patients and families with information that will help them in their decision-making regarding treatment of bowel obstruction. In the presence of bowel obstruction, patients and families have a significant amount of informational needs around the cause of bowel obstruction, options for management, and supportive care available. The 'best' management for bowel obstruction has not yet been established, therefore, nurses must help patients/families explore short- and longer-term goals. Nurses must also communicate these decisions to the multidisciplinary team in order to ensure that a treatment plan is established that is based on the patient's and her family's goals. Because bowel obstruction is often the first sign of recurrent disease, support and information around recurrence and prognosis contributes to the complexity of care and must be provided.

In managing patients with a bowel obstruction using a PEG tube, nurses play a large part in assessing the patient's symptoms on an ongoing basis. A significant percentage of nursing care will need to be spent on helping the patient and family learn about the care of the PEG tube, and ongoing assessment of whether the PEG tube should be to gravity, suction, or clamped. In this study, data on whether the PEG tube was to suction, gravity, or clamped were available in only $50 \%$ of the patients. Therefore, it is difficult to draw conclusions directly from the study. Further, the description of symptoms and their management was documented in several areas of the chart with no consistent method. In order to improve nursing care and multidisciplinary communication, documentation of symptoms related to bowel obstruction, such as nausea, vomiting, pain, bowel function, and PEG tube management, needs to be improved. This will facilitate the tailoring of interventions including concomitant medications used as adjuvants to the PEG. Consequently, we have 
identified the need to develop a tool that will help nurses in the assessment of patients and in implementation of interventions to address those needs. There are plans to develop a working group to identify the most appropriate tool, e.g., standard care plans, medical directives, flowsheets, or intervention guidelines.

The mean length of stay in our study was 15.1 days. More research is needed to determine if the length of stay could be optimized by improved discharge planning or if perhaps the implications of bowel obstruction are too overwhelming to patients and their families. However, the mean time from admission to PEG was 8.1 days and $50 \%$ of the patients in our study had one or two prior admissions for bowel obstruction. There is an opportunity to initiate counselling around PEG during the first admission for bowel obstruction, even when patients have a spontaneous resolution, as the literature suggests that $32 \%$ to $45 \%$ of patients will experience a re-obstruction (Feuer et al., 2000).

\section{Conclusion}

Oncology nurses who care for women with ovarian cancer and their families must have a good understanding of malignant bowel obstruction, its treatment options, and their limitations. This

\section{References}

Abu-Rustum, N., Barakat, R.R., Venkatramann, E., \& Spriggs, D. (1997). Chemotherapy and total parenteral nutrition for advanced ovarian cancer with bowel obstruction. Gynecologic Oncology, 64, 493-495.

Adelson, M.D., \& Kasowitz, M.H. (1993). Percutaneous endoscopic drainage gastrostomy in the treatment of gastrointestinal obstruction from intraperitoneal malignancy. Obstetrics \& Gynecology, 81(3), 467-471.

Baumrucker, S. (1998). Management of intestinal obstruction in hospice care. The American Journal of Hospice \& Palliative Care, 232-235.

Beattie, G., Leonard, R.C.F., \& Smyth, J.F. (1989). Bowel obstruction in ovarian carcinoma: A retrospective study and review of the literature. Palliative Medicine, 3, 275-280.

Cain, J., Wenzel, L., Monk, B., \& Cella, D. (1998). Palliative care and quality of life. In D.M. Gershenson \& W.P. McGuire (Eds.), Ovarian cancer: Controversies in management (pp. 281-303). Edinburgh, Scotland: Churchill Livingston.

DiScia, P.J., \& Creasman, W.T. (2002). Epithelial ovarian cancer. Clinical gynecologic oncology (pp. 289-343). St. Louis, MO: Mosby.

Fainsinger, R.L., Spachynski, K., Hanson, J., \& Bruera, E. (1994). Symptom control in terminally ill patients with malignant bowel obstruction. Journal of Pain and Symptom Management, 9(1), 12-18.

Feuer, D.J., Broadley, K.E., \& Barton, D.P.J. (2000). Surgery for the resolution in malignant bowel obstruction in advanced gynecological and gastrointestinal cancer. Cochrane Database of Systematic Reviews, 4, CD002764.

Frankel Kelvin, J., \& Scagliola, J. (1998). Metastases involving the gastrointestinal system. Seminars in Oncology Nursing, 14(3), 187-198

Gadducci, A., Iacconi, I., Fanucchi, A., Cosio, S., Miccoli, P., \& Genazzani, A.R. (1998). Survival after intestinal obstruction in patients with fatal ovarian cancer: Analysis of prognostic variables. International Journal of Gynecological Cancer, 8, 177-182.

Gemlo, B., Rayne, A.A., Lewis, B., Wong, A., Viele, C.S., Ungaretti, J.R., et al. (1986). Home support of patients with end-stage malignant bowel obstruction using hydration and venting gastrostomy. The American Journal of Surgery, 152, 100-104. quality improvement study indicates that successful symptom control can be achieved using a PEG tube in bowel obstruction with recurrent ovarian cancer, and has helped to identify opportunities to improve the care provided to these women treated at our institution.

Research is needed to gain a better understanding of the impact that bowel obstruction has on women with ovarian cancer, and to identify the best option for palliation of bowel obstruction in ovarian cancer. Quality of life measurement needs to be included in research, and outcome measurements need to be standardized. In their Cochrane review, Feuer et al. (2000) recommended that nausea, vomiting, pain, and constipation be measured as outcome indicators of surgery for bowel obstruction. The same outcomes need to be measured with all types of management, not just PEG, in order to determine the best options. Other questions that must be investigated are: "What is the impact of hydration on symptom control in palliation of bowel obstruction from a physical and psychosocial perspective?" and "Is chemotherapy beneficial in palliating patients whose goals are to maximize time?"

Hopkins, M.P., Roberts, J.A., \& Morley, G.W. (1987). Outpatient management of small bowel obstruction in terminal ovarian cancer. The Journal of Reproductive Medicine, 32(11), 827-829.

Jong, P., Sturgeon, J., \& Jamieson, C.G. (1995). Benefit of palliative surgery for bowel obstruction in advanced ovarian cancer. Canadian Journal of Surgery, 5, 454-457.

Kehoe, S., \& Mann, C. (2002). Role of surgery in relapse and palliation of ovarian cancer. In I. Jacobs, J. Shepherd, D. Oram, A. Blackett, D. Luesley, A. Berchuck, \& C. Hudson (Eds.), Ovarian cancer, (pp. 305-309). Oxford, England: Oxford University Press.

Khoo, D., Hall, E., Motson, R., Riley, J., Denman, K., \& Waxman, J. (1994). Palliation of malignant intestinal obstruction using Octriotide. European Journal of Cancer, 30A, 28-30.

Malone, J.M., Koonce, T., Larson, D.M., Freedman, R.S., Carrasco, C.H.O., \& Saul, P.B. (1986). Palliation of small bowel obstruction by percutaneous gastrostomy in patients with progressive ovarian carcinoma. Obstetrics \& Gynecology, 68(3), 431-433.

Mangili, G., Franchi, M., Mariani, A., Zanaboni, F., Rabaitti, E., Frigerio, L., et al. (1996). Octriotide in the management of bowel obstruction in terminal ovarian cancer. Gynecologic Oncology, 61, 345-348.

Marks, W., Perkal, M.F., \& Schwartz, P.E. (1993). Percutaneous endoscopic gastrostomy for gastric decompression in metastatic gynecologic malignancies. Surgery, Gynecology and Obstetrics, 177, 573-576

Mercandante, S., Spoldi, E., Caraceni, A., Maddaloni, S., \& Simonetti, M.T. (1993). Octriotide in relieving gastrointestinal symptoms due to bowel obstruction. Palliative Medicine, 7, 295-299.

National Cancer Institute of Canada. (2002). Canadian cancer statistics 2002. Toronto: Author.

Ripomonti, C. (1994). Management of bowel obstruction in advanced cancer. Current Opinion in Oncology, 6, 351-357.

van Ooijen, B., van der Burg, M.E.L., Planting, A.S.Th., Siersema, P.D., \& Wiggers, T. (1993). Surgical treatment or gastric drainage only for intestinal obstruction in patients with carcinoma of the ovary or peritoneal carcinomatosis of other origin. Surgery, Gynecology and Obstetrics, 176, 469-474. 